\title{
STURM COMPARISON THEOREMS FOR ELLIPTIC INEQUALITIES
}

\author{
BY W. ALLEGRETTO AND C. A. SWANSON ${ }^{1}$ \\ Communicated by M. H. Protter, May 21, 1969
}

Comparison theorems of Sturm's type will be stated for the quasilinear elliptic partial differential inequalities

$$
\begin{aligned}
& \text { (1) } \quad l u=-\sum_{i, j=1}^{n} D_{i}\left[a_{i j}(x, u) D_{j} u\right]+2 \sum_{i=1}^{n} b_{i}(x, u) D_{i} u+u c(x, u) \leqq 0, \\
& \text { (2) } \quad L v=-\sum_{i, j=1}^{n} D_{i}\left[A_{i j}(x, v) D_{j} v\right]+2 \sum_{i=1}^{n} B_{i}(x, v) D_{i} v+v C(x, v) \geqq 0, \\
& x=\left(x_{1}, \cdots, x_{n}\right) \in G, \quad u, v \in I, \quad D_{i}=\partial / \partial x_{i} \quad(i=1, \cdots, n)
\end{aligned}
$$

where $G$ is a nonempty regular bounded domain in $R^{n}$ and $I$ is a real interval containing zero. The functions $a_{i j}, A_{i j}, b_{i}, B_{i}, c$, and $C$ are assumed to be real-valued and continuous on $\bar{G} \times I$, and the matrices $\left(a_{i j}\right)$ and $\left(A_{i j}\right)$ symmetric and positive definite in $G \times I$.

A Sturmian theorem has the following form: If (1) has a nontrivial solution $u$ which vanishes identically on the boundary of $G$ and if (2) majorizes (1) in some sense, then every solution $v$ of (2) has a zero in $\bar{G}$ (or $G$ ).

The linear selfadjoint case $\left(b_{i}=B_{i}=0, i=1, \cdots, n\right)$ was first considered by Picone [12], and later independently and more generally by Hartman and Wintner [4], Kuks [10], Kreith [6], [8], Clark and Swanson [2]. A recent research announcement of Diaz and McLaughlin [3] is similar to Kreith's "strong comparison theorem" [9], obtained when $\partial G$ has the "sphere property" by an appeal to the Hopf maximum principle. The conclusion of the strong comparison theorem is that $v$ has a zero in $G$ unless $v$ is a constant multiple of $u$; an analogous result in the quasilinear case is stated below (Theorem 2). Earlier $\mathrm{McNabb}$ [11] had used similar techniques in a different connection.

The linear nonselfadjoint case was studied by Protter [13], Swanson [16], Kreith [9], and Allegretto [1]. Extensions to unbounded domains were obtained in [16] and [17] and applied to oscillation theory and eigenvalue estimation. Comparison theorems

1 Research sponsored by the Air Force Office of Scientific Research, Office of Aerospace Research, United States Air Force, under AFOSR Grant No. AFOSR-681531. 
in terms of eigenvalues associated with boundary problems for differential operators also have been developed [7], [8], [15] and used to derive oscillation criteria [1], [5]. The quasilinear case was considered by Redheffer [14] and the authors [1], [19]. An extensive bibliography on comparison and oscillation theory can be found in [18].

Let $m, M$ denote the $(n+1)$-square matrix functions on $\bar{G} \times I$ defined by

$$
\begin{aligned}
m(x, u) & =\left(\begin{array}{cc}
\left(a_{i j}(x, u)\right) & \left(b_{i}(x, u)\right)^{T} \\
\left(b_{i}(x, u)\right) & c(x, u)
\end{array}\right), \\
M(x, u) & =\left(\begin{array}{cc}
\left(A_{i j}(x, u)\right) & \left(B_{i}(x, u)\right)^{T} \\
\left(B_{i}(x, u)\right) & C(x, u)+H(x, u)
\end{array}\right)
\end{aligned}
$$

respectively, where

$$
H(x, u)=-\left[\operatorname{det}\left(A_{i j}(x, u)\right)\right]^{-1} \sum_{i=1}^{n} B_{i}(x, u) B_{i}^{*}(x, u),
$$

$B_{i}^{*}(x, u)$ denoting the cofactor of $B_{i}(x, u)$ in the matrix $M(x, u)$.

By means of Green's formula the following functional is associated with $l$ in a natural way

$$
\begin{aligned}
f[u]= & \int_{G}\left[\sum_{i, j} a_{i j}(x, u(x)) D_{i} u D_{j} u+2 u \sum_{i} b_{i}(x, u(x)) D_{i} u\right. \\
& \left.+u^{2} c(x, u(x))\right] d x .
\end{aligned}
$$

The domain $\mathfrak{D}$ of $f$ is defined to be the set of all real-valued functions $u \in C^{1}(\bar{G})$ with range in $I$ such that $u$ vanishes identically on $\partial G$.

THEOREM 1. If

(1) there exists a function $u \in \mathbb{D}$ such that $f[u] \leqq 0$ (respectively, $f[u]<0)$;

(2) $L v \geqq 0$ throughout $G$;

(3) $v(x)>0$ for some $x \in G$;

(4) $m(x, u(x))-M(x, v(x))$ is positive definite (respectively, positive semidefinite) for all $x \in G$;

then $v$ must vanish at some point in $\bar{G}$. The same conclusion holds if the inequalities in (2) and (3) are replaced by Lv $\leqq 0$ and $v(x)<0$. The same conclusion holds if (2) and (3) are replaced by $L v=0$ throughout $G$.

It follows from Green's formula that hypothesis (1) is implied by the existence of a solution $u$ of $l u \leqq 0$ (respectively, $l u \geqq 0$ ) satisfying $u>0$ (respectively, $u<0$ ) throughout $G$ and $u=0$ on $\partial G$. 
The "strong" conclusion that $v$ must in fact vanish at some point in $G$ can be obtained by our methods [1], [19] under additional assumptions. Also, an analogue of Theorem 1 is available when the coefficients $a_{i j}, b_{i}, c$, etc. are functions of first or higher order derivatives of $u$. These results with proofs will appear elsewhere.

Of special interest in oscillation theory are cases for which the hypotheses of Theorem 1 are satisfied when $l$ is linear. In such cases, the known properties of linear symmetric operators can be employed to describe the oscillatory behaviour of $L$. Simple examples which can be treated this way are Mathieu's and Duffing's equations.

The pointwise inequality in hypothesis (4) of Theorem 1 can be replaced by a weaker integral inequality of the type given in [2], [15], [16], and [17]. For simplicity, we shall state our result in the selfadjoint case $b_{i}=B_{i}=0$ identically, $i=1, \cdots, n$. Let $F[u]$ denote the analogue of the functional (3) for $L$, i.e. with $a_{i j}$ and $c$ in (3) replaced by $A_{i j}$ and $C$, respectively.

Theorem 2 (SelfadjoInt CASE). Suppose that $L$ is uniformly elliptic in a nonempty regular bounded domain $G$ whose boundary has bounded curvature, and that the matrix function $v \rightarrow M(x, v)$ is nonincreasing (as a form) on I for each $x \in G$. If there exists a nontrivial solution $u \in \mathfrak{D}$ of (1) such that $u>0$ in $G$ and $f[u] \geqq F[u]$, then every solution $v$ of (2) has one of the following properties:

(i) There exists a subdomain $G_{v} \subset G$ such that $v(x)<u(x)$ for all $x \in G_{v}$, or

(ii) $v$ is a constant multiple of $u$.

An example given in [19] shows that the conclusion of Theorem 2 is false without the nonincreasing hypothesis on $M$. In the linear case, conclusion (i) is strengthened to

(i') $v(x)$ vanishes at some point $x \in G$ (Kreith's theorem [8]). Theorem 2 can be used to obtain nonoscillation criteria of the KneserHille-Glazman type [19].

\section{REFERENCES}

1. W. Allegretto, Comparison and oscillation theorems for elliptic equations, Ph.D. thesis, University of British Columbia, Vancouver, British Columbia, Canada, 1969.

2. Colin Clark and C. A. Swanson, Comparison theorems for elliptic differential equations, Proc. Amer. Math. Soc. 16 (1965), 886-890.

3. J. B. Diaz and Joyce R. McLaughlin, Sturm comparison theorems for ordinary and partial differential equations, Bull. Amer. Math. Soc. 75 (1969), 335-339.

4. Philip Hartman and Aurel Wintner, On a comparison theorem for self-adjoint partial differential equations of elliptic type, Proc. Amer. Math. Soc. 6 (1955), 862-865. 
5. V. B. Headley and C. A. Swanson, Oscillation criteria for elliptic equations, Pacific J. Math. 27 (1968), 501-506.

6. Kurt Kreith, $A$ new proof of a comparison theorem for elliptic equations, Proc. Amer. Math. Soc. 14 (1963), 33-35.

7. - Comparison theorems for constrained rods, SIAM Rev 6 (1964), 31-36.

8. - A strong comparison theorem for selfadjoint elliptic equations, Proc. Amer. Math. Soc. 19 (1968), 989-990.

9. - A remark on a comparison theorem of Swanson, Proc. Amer. Math. Soc. 20 (1969), 549-550.

10. L. M. Kuks, Sturm's theorem and oscillation of solutions of strongly elliptic systems, Soviet Math. Dokl. 3 (1962), 24-27.

11. A. McNabb, Strong comparison theorems for elliptic equations of second order, J. Math. Mech. 10 (1961), 431-440.

12. Mauro Picone, Un teorema sulle soluzioni delle equazioni lineari ellittiche autoaggiunte alle derivate parziali del secondo-ordine, Rend. Acc. Lincei 20 (1) (1911), 213-219.

13. M. H. Protter, A comparison theorem for elliptic equations, Proc. Amer. Math. Soc. 10 (1959), 296-299.

14. R. M. Redheffer, A Sturmian theorem for partial differential equations, Proc. Amer. Math. Soc. 8 (1957), 458-462.

15. C. A. Swanson, A generalization of Sturm's comparison theorem, J. Math. Anal. Appl. 15 (1966), 512-519.

16. - Comparison theorems for elliptic equations on unbounded domains, Trans. Amer. Math. Soc. 126 (1967), 278-285.

17. - An identity for elliptic equations with applications, Trans. Amer. Math. Soc. 134 (1968), 325-333.

18. - Comparison and oscillation theory of linear differential equations, Mathematics in Science and Engineering, vol. 48, Academic Press, New York, 1968.

19. - Comparison theorems for quasilinear elliptic differential inequalities, J. Differential Equations (to appear).

University of British Columbia, Vancouver, British Columbia, Canada 